\title{
TOPOGRAPHIC MAPPING OF THE MOON IN THE 21ST CENTURY: FROM HECTOMETER TO MILLIMETER SCALES
}

\author{
K. $\mathrm{Di}^{1}{ }^{1}$, J. Oberst ${ }^{2}$, I. Karachevtseva ${ }^{3}$, B. $\mathrm{Wu}^{4}$ \\ ${ }^{1}$ State Key Laboratory of Remote Sensing Science, Aerospace Information Research Institute, \\ Chinese Academy of Sciences, Beijing, China - dikc@radi.ac.cn \\ ${ }^{2}$ German Aerospace Center, Institute of Planetary Research, Berlin, Germany - Juergen.Oberst@dlr.de \\ ${ }^{3}$ Moscow State University of Geodesy and Cartography (MIIGAiK), Moscow, Russia - i_karachevtseva@miigaik.ru \\ ${ }^{4}$ Department of Land Surveying and Geo-Informatics, The Hong Kong Polytechnic University, Hung Hom, Kowloon, Hong Kong - \\ bo.wu@polyu.edu.hk
}

\section{Commission III, ICWG III/II}

KEY WORDS: Lunar Exploration, Topographic Mapping, Photogrammetry, Orbital Imagery, Descent Imagery, Rover-based Imagery

\begin{abstract}
:
This paper presents a review of lunar topographic mapping in the two decades of the $21^{\text {st }}$ century, including descriptions of lunar exploration missions, relevant payloads and data, mapping techniques, as well as global and regional mapping products. Various lunar photogrammetric mapping techniques such as construction of geometric models of lunar orbital images, block adjustments, shape from shading, co-registration of lunar orbital image and elevation data have been developed to process lunar orbital images and generate mapping products. Global topographic products at hectometer and decameter scales have been produced from orbital images and/or laser altimeter data. Regional topographic maps of the landing sites and other sites of interest have been generated at meter-scale using the sub-meter to meter resolution orbital images. Detailed local topographic products at centimeter to millimeter scales of the landing sites and rover traverse areas have been produced using descent images acquired by the landers and stereo images acquired by the rovers. These multiple-scale topographic mapping products have been extensively used to support various science applications, as well as engineering applications such as surface operations of the rovers.
\end{abstract}

\section{INTRODUCTION}

The first two decades of the $21^{\text {st }}$ century have seen an increasing number of lunar exploration missions, including orbital missions and landed rover missions. The orbital missions, using the onboard cameras and laser altimeters, have collected unprecedented images and laser altimetry data in terms of resolution, accuracy, and data volume. While orbital observations provide global and regional information of the lunar surface, the rover missions offer detailed in-situ investigations of the landing sites.

Topographic mapping is one of the fundamental tasks in most lunar exploration missions (Greeley and Batson, 1990; Kirk et al., 2008, 2012; Di et al., 2015; Naß et al., 2017; Wu et al., 2019). It is usually conducted based on photogrammetric techniques using the data acquired by orbiters, landers, and rovers at hectometer to millimeter scales. The mapping products, such as digital terrain models (DTMs) and digital orthophoto maps (DOMs), have greatly contributed to exploration and scientific research of the Moon. For example, the topographic mapping products from orbital images are the bases for morphological analyses of lunar landforms, and are critical to support lunar geologic studies. Meanwhile, high-resolution orbital mapping products are particularly important to support landing site evaluation for a lander/rover mission, and also valuable to support lander localization, science target designation, path planning and rover localization ( $\mathrm{Wu}$ et al., 2014a, 2020; Liu et al., 2015, 2020; Di et al., 2019a, 2020).

In principle, the photogrammetric techniques used for lunar topographic mapping are similar to that used in earth observation applications. However, the precisions of orbit and attitude of lunar orbiters are much lower than that of earth satellites. It is very hard to obtain high-accuracy ground control points on the Moon. There is no global navigation satellite system (GNSS) available on the Moon orbit. Moreover, the lunar imagery is severely affected by changing illumination conditions due to lack of atmosphere. These factors bring great challenges to lunar photogrammetry. New developments have been made to overcome the challenges so that to generate various mapping products with improved accuracy and higher resolution.

This paper presents a review of lunar topographic mapping in the two decades of the $21^{\text {st }}$ century, including descriptions of lunar exploration missions, relevant payloads and data, mapping techniques, as well as global and regional mapping products.

\section{LUNAR EXPLORATION MISSIONS AND RELEVANT DATA}

From 2003 to 2019, ten lunar orbital missions, including SMART-1, SELENE, Chang'e-1, Chandrayaan-1, Lunar Reconnaissance Orbiter (LRO), Chang'e-2, ARTEMIS, Gravity

\footnotetext{
* Corresponding author
} 
Recovery and Interior Laboratory (GRAIL), Lunar Atmosphere and Dust Environment Explorer (LADEE), and Chandrayaan-2 were successfully launched and orbited the Moon for various mission tasks. The orbital imagers acquired hectometer to meter level resolution images. Two lander and rover missions, Chang'e-3 and Chang'e-4, were successfully landed on the Moon and provided in-situ observations of the landing sites with image resolutions from better than millimeter level to centimetre level. As the imaging cameras and laser altimeters are the major instruments for topographic mapping of the lunar surface. We only briefly summarize the missions that carried cameras and/or laser altimeters below.

SMART-1 (Small Missions for Advanced Research in Technology-1) was launched by European Space Agency (ESA) in September 2003, successfully entered lunar orbit in November 2004, and finally conducted a controlled impacting on the lunar surface in September 2006. SMART-1's Advanced Moon Micro-imager Experiment (AMIE) acquired CCD images with an average spatial resolution of $80 \mathrm{~m}$, and a perilune (300 $\mathrm{km}$ ) spatial resolution of $30 \mathrm{~m}$ (Foing et al., 2006). Totally more than 32,000 images were acquired covering the entire Moon with a resolution better than $250 \mathrm{~m}$ per pixel and covering the southern hemisphere at about $100 \mathrm{~m}$ per pixel (Grieger et al., 2008).

The Japanese SELENE (SELenological and Engineering Explorer) mission, also known as Kaguya, was launched in September 2007, began operations in December, and conducted a controlled impacting on the lunar surface in June 2009. SELENE's terrain camera (TC) acquired stereo images through push-broom imaging with a resolution of $10 \mathrm{~m}$ with nearly global coverage at a circular orbit of $100 \mathrm{~km}$ (Haruyama et al., 2012). The laser altimeter (LALT) collected global altimetry data with $1.6 \mathrm{~km}$ along track spacing and $5 \mathrm{~m}$ vertical resolution (Araki et al., 2008).

Chang'e-1 (CE-1) was China's first lunar orbiter. It was launched in October 2007, entered the orbit $200 \mathrm{~km}$ above the lunar surface and began science exploration in November, and ended with a controlled impacting in March 2009. CE-1's threeline CCD camera acquired forward-, nadir- and backwardlooking images of the entire moon with a spatial resolution of $120 \mathrm{~m}$. The laser altimeter (LAM) collected global altimetry data with a range resolution of $1 \mathrm{~m}$. The CE-1 detector operated in orbit for 495 days, and obtained 1.39 TB of raw scientific data through which $4 \mathrm{~TB}$ of scientific application data was produced (Zuo et al., 2014).

The Indian Chandrayaan-1 mission was launched in October 2008, and ended its operation in August 2009. The three-line terrain mapping camera (TMC) conducted stereoscopic imaging with a spatial resolution of $5 \mathrm{~m}$. The lunar laser ranging instrument (LLRI) had a range resolution of better than $5 \mathrm{~m}$, with its data covering the polar regions of the Moon (Goswamiet and Annadurai, 2009).

The U. S. LRO mission was launched by NASA in June 2009 . LRO initially entered an oval-shaped test run orbit after four days of flight, and then entered a circular polar orbit $50 \mathrm{~km}$ from the lunar surface. As of May 2020, the LRO mission is still on operation. The lunar reconnaissance orbiter camera (LROC) consists of a wide-angle camera (WAC) and two narrow-angle cameras (NAC) (Robinson et al., 2010). WAC acquires images with a spatial resolution of $100 \mathrm{~m}$ and a swath of $100 \mathrm{~km}$, NAC acquires images with a spatial resolution of
$0.5-2 \mathrm{~m}$ and a swath of $5 \mathrm{~km}$. The Lunar orbiter laser altimeter (LOLA) acquires 5 parallel profiles, separated by $\sim 56 \mathrm{~m}$, with short intervals of $\sim 10-12 \mathrm{~m}$ along the track, and a range resolution of $10 \mathrm{~cm}$ (Smith et al., 2010a).

China's Chang'e-2 (CE-2) orbiter was launched in October 2010. The two-line CCD camera acquired stereo images with a spatial resolution of $7 \mathrm{~m}$ and $1.05 \mathrm{~m}$ at the orbit altitudes of 100 $\mathrm{km}$ and $15 \mathrm{~km}$, respectively. By completion of the mission, the CE-2 CCD camera obtained 607 orbits of image data, with $7 \mathrm{~m}$ resolution images covering the entire Moon and $1.05 \mathrm{~m}$ resolution images covering the preselected landing area of Chang'e-3 (Zuo et al., 2014).

Chang'e-3 (CE-3) was the third mission in China's Chang'e lunar exploration program and the first soft landing mission. The probe, including a lander and a rover, was launched on 2 December 2013, and successfully landed in northern Mare Imbrium of the moon on 14 December 2013 (Wu et al., 2014a). The Yutu (Jade Rabbit) rover was released to the lunar surface and began surface exploration on December 15. The rover traversed $114.8 \mathrm{~m}$ on the lunar surface and accomplished a series of scientific investigations under teleoperation (Liu et al., 2015).

Chang'e-4 (CE-4) mission was designed to be a backup of the CE-3 mission. It was launched on 8 December 2018. With the support of the Queqiao (Magpie Bridge) relay satellite, CE-4 successfully landed on the far side of the moon in Von Kármán crater on 3 January 2019 (Di et al., 2019b; Wu et al., 2020). The Yutu-2 rover was successfully released from the lander and began surface exploration on the same day. The success of CE4 marks the first soft landing of human spacecraft on the far side of the moon. By the end of the $12^{\text {th }}$ lunar day (As of December 4, 2019), the rover has traversed $345.059 \mathrm{~m}$ (China Daily, 2019).

Both CE-3 and CE-4 landers carry a downward-looking descent camera with a field of view (FOV) of $45.4^{\circ}$ and an image size of $1024 \times 1024$ pixels (Liu et al. 2015). The descent camera took thousands of images (about 4600 in CE-3 and 5300 in CE-4 mission) with resolutions from meter level to millimeter level during the entry, descent, and landing (EDL) process.

Both Yutu and Yutu-2 rovers carry three pairs of stereo cameras, i.e., panoramic camera (Pancam), navigation camera (Navcam), and hazard avoidance camera (Hazcam). Pancam and Navcam are mounted on the same camera bar atop the rover mast; Hazcam is fixed on the lower front of the rover body. Both Pancam and Navcam have a stereo base of $270 \mathrm{~mm}$. The Pancam image is of 2352 pixels $\times 1728$ pixels with the FOV of $22.9^{\circ} \times 16.9^{\circ}$ (Jia et al. 2018). The Navcam image is of 1024 $\times 1024$ pixels with the FOV of $46.6^{\circ}$ (Liu et al. 2015). Along the traverse in each mission, the rover obtains Navcam stereo images at every waypoint and acquires Pancam stereo images at some waypoints. These stereo images, with better than millimeter resolutions in near-range and centimeter resolutions in middle-range, have been routinely used in detailed topographic mapping and rover localization to support mission operations (Liu et al., 2020; Di et al., 2020).

In addition to optical images and laser altimetry data, radar data, such as images collected by Mini-SAR onboard Chandrayaan1 and Mini-RF onboard LRO, were also used to derive DTMs of the moon (Kirk et al., 2010). 


\section{RECENT PROGRESS ON LUNAR TOPOGRAPHIC MAPPING TECHNIQUES}

\subsection{Geometric Modelling of Lunar Images}

Photogrammetric techniques have been developed for topographic mapping using the new orbital, descent, and rover data (Kirk et al., 2008, 2012; Oberst et al., 2014; Wu et al., 2019; Di et al., 2020). Rigorous geometric modelling of the lunar images is the basis for high precision geometric processing of the images. Rigorous geometric models (RSMs) of lunar orbital images are construed based on collinearity equations with interior orientation (IO) parameters and exterior orientation (EO) parameters (Tran et al., 2010; Haruyama et al., 2012; Di et al., 2014; Speyerer et al., 2016; Wu et al., 2014; Wu and Liu, 2017).

To obtain the IO parameters of the camera, camera calibration and interior orientation are necessary and are usually done preflight and sometimes on-orbit (Speyerer et al., 2016; Wu and Liu, 2017). EO parameters are derived from orbit and attitude measurements, the accuracies of which have been significantly improved in the $21^{\text {st }}$ century. For example, benefiting from gravity field data from the GRAIL mission and crossovers from LOLA data, LRO orbit determination has reached a high accuracy of $\sim 14 \mathrm{~m}$ (Mazarico et al., 2012). Orbital images are usually obtained using push-broom imaging principle. Thus, the EO parameters of push-broom orbital imagers are timedependent, i.e., each image scan line has a specific set of EO parameters. The changes of the EO parameters over short trajectories are usually modelled by third-order polynomials ( $\mathrm{Di}$ et al., 2014).

In addition to RSM, the rational function model (RFM) has also been evaluated and used in photogrammetric processing of planetary orbital images (Liu and Di, 2011; Liu et al., 2014, 2016; $\mathrm{Hu}$ and $\mathrm{Wu}, 2018,2019)$. The RFM model parameters of an orbital image, i.e., the rational polynomial coefficients (RPCs), are obtained by least-squares fitting with a large number of virtual control points generated by the RSM of the image. The RFM can approximate the RSM at a precision of $1 / 100$ pixel in image space for orbiters without exposure time changing (e.g., CE-1, LROC NAC, HiRISE). It is worth noting that for orbiters with exposure time changing (e.g., CE-2 and HRSC), Liu et al. (2016) proposed two solutions, time-based RFM or sensor corrected images with line-based RFM, to reach such a high fitting precision. The RFM has the advantages of simple and uniform form, and imaging sensor independence. It is particularly convenient in combined photogrammetric processing of images from multi-missions (Di et al., 2017).

The RSMs of lander and rover images are also established based on collinearity equations with IO and EO parameters. The descent camera, Pancam, Navcam, and Hazcam are all frame cameras; thus, the whole image has one set of EO parameters. To ensure high precision measurement, the fixed relative orientation of the left image and right image of the stereo camera were calibrated along with the IO parameters and enforced in the subsequent photogrammetric processing (Peng et al., 2014; Wan et al., 2014).

\subsection{Photogrammetric Block Adjustment for Geometric Model Refinement}

Due to various uncertainties in orbit and attitude measurements, timing, and image calibration, etc., the established RSMs or
RFMs are not error-free. Directly using the original RSMs or RFMs usually result in positioning errors of lunar surface points and inconsistencies among images of different missions and among neighboring-track images of the same mission. Photogrammetric block adjustment is usually employed to refine the geometric models so that to remove or reduce these positioning errors and inconsistencies;

Block adjustment is a powerful tool to improve the positioning and mapping accuracies of the images by solving the EO parameters and 3-D ground points simultaneously using tie points that link the images together. Different strategies and methods have been developed by different teams to process the orbital images of different missions. The block adjustment can be either based on RSM (also called bundle adjustment) or RFM. In general, block adjustment of lunar orbital images in a local area can achieve a sub-pixel precision.

Haruyama et al. (2009) corrected the offset of instrument view vectors among multiple SELENE TC images and reduced the relative difference from $\sim 100 \mathrm{~m}$ to better than $10 \mathrm{~m}$, subsequently $10 \mathrm{~m}$-resolution uncontrolled DTMs were produced globally. To improve the accuracy of TC DTMs, Haruyama et al. (2012) corrected the distortion models and attachment angles of TC using the LALT data as vertical control, decreasing the differences between TC DTMs and LALT measurements to $3.2 \mathrm{~m}$.

Radhadevi et al. (2013) developed a bundle block adjustment algorithm for geometric correction of Chandrayaan-1 TMC images based on RSM, using LOLA data as vertical control, and using $100 \mathrm{~m} /$ pixel USGS Clementine base map as planimetric control. The inconsistencies among images were reduced and root-mean-square (RMS) error of the order 200 $300 \mathrm{~m}$ in latitude, longitude and height with respect to the references was achieved.

Although the LROC is not designed with built-in stereo capability, NAC stereo images can be acquired from adjacent orbits using off-nadir slew for high-resolution DTM and DOM production. Photogrammetric modelling and processing of LROC NAC images has been performed by several teams in the LRO mission, e.g., Arizona State University (ASU), German Aerospace Center (DLR), Technische Universität Berlin (TUB), National Aeronautics and Space Administration (NASA) Ames Research Center, University of Arizona (UA), Ohio State University (OSU), and United States Geological Survey (USGS), using ISIS and SOCET SET or other in-house developed software (Tran et al., 2010; Li et al., 2010). Bundle block adjustment was one of the key techniques in the processing and evaluation of LROC NAC images. Through bundle adjustment of stereo images, the RMS errors can be reduced to sub-pixel to one pixel level (Henriksen et al., 2017; Haase et al., 2019). So far, LROC NAC stereo images have been widely used in generation of high-resolution mapping products after block adjustment for various applications, e.g., landing site topographic analysis (Haase et al., 2012; Karachevtseva et al., 2013; Wu et al., 2014a).

RSMs of CE-1 and CE-2 images have been developed and used in block adjustments in several ways. For example, combined adjustment methods for the CE-1 imagery and laser altimeter, and for CE-2 imagery and LOLA data have been developed respectively to reduce the inconsistencies among images to 1 pixel to sub-pixel level (Wu et al., 2011; Wu et al., 2014b). A method for CE-1 RSM model refinement was developed 
through co-registration of CE-1 stereo images and laser altimeter data with crossover adjustment (Di et al., 2012). A self-calibration bundle adjustment method has been developed for CE-2 images by introducing additional IO parameters in the bundle adjustment, which reduced the back projection residuals of neighboring tracks from 20 pixels to sub-pixel (Di et al., 2014). RFM based block adjustment methods have also been developed and achieved sub-pixel precision as well (Liu et al., 2014; $\mathrm{Hu}$ and $\mathrm{Wu}, 2018$ ). Ren et al. (2019) developed a global adjustment method for photogrammetric processing of Chang'E-2 stereo images, and the deviations between the neighboring strips were reduced to sub-pixel level.

The block adjustment methods are usually applied to stereo images. To support high-resolution large-area mapping using single images, e.g., LROC NAC images, Di et al. (2019c) developed a DTM-assisted 2-D block adjustment for highresolution large-area DOM generation, based on which a seamless DOM of the pre-selected Chang'e-5 landing area was produced with a resolution of $1.5 \mathrm{~m}$ using over 700 LROC NAC images.

\subsection{Mapping Product Generation}

The standard procedure of topographic mapping from stereo lunar images include establishment of geometric models of the involved images, sparse image matching for tie point selection, block adjustment, dense image matching, 3-D dense points calculation by space intersection with adjusted image model parameters, DTM generation by interpolation, and DOM generation through back-projection and resampling.

Many software packages have been developed by different organizations and groups specifically for lunar and planetary mapping, within which DTM and DOM production is the key components (e.g., Moratto et al., 2010; Beyer et al., 2018; Re et al., 2012; Kozlova et al., 2014; Liu et al., 2015, 2020, Karachevtseva et al., 2016; Zubarev et al., 2016; $\mathrm{Hu}$ and $\mathrm{Wu}$, 2019). If the RPCs are provided along with the lunar orbital images, commercial photogrammetric software systems, which are basically used for earth observations, can be used conveniently to produce DTMs of the lunar surface. To a large extent, the DTM quality depends on image matching results. Recently, the semi-global matching (SGM) algorithm (Hirschmuller, 2007) has gained popularity due to its good performance and widely adopted in the development of lunar mapping systems.

In recent years, in addition to techniques in the standard procedure for topographic mapping from stereo orbital images, other relevant techniques have also been developed for various lunar mapping scenarios and applications, e.g., co-registration of multiple source DTM and imagery data (Wu et al., 2013; Xin et al., 2018), generation of pixel-level resolution DTMs from monocular images by shape-and-albedo-from-shading constrained with low-resolution DTM (Wu et al., 2017), integrated photogrammetric and photoclinometric approach for pixel-level 3D reconstruction of the lunar surface from images with different illumination conditions (Liu and $\mathrm{Wu}, 2020$ ), and progressive selection of best image combinations for achieving highest 3-D positioning precision in areas with multiple image coverages (Liu et al. 2018), etc.

\section{TOPOGRAPHIC MAPPING PRODUCTS}

\subsection{Global Mapping Products}

Multiple global DTMs have been produced from optical stereo images or laser altimetry data acquired in the $21^{\text {st }}$ century. Global DOMs have also been produced from orbital images. These global DTMs and DOMs are publically available and have been extensively used in lunar topographic, morphologic, and geologic studies.

The laser altimetry data from SELENE (Araki et al., 2009), Chang'e-1 (Ping et al., 2008; Li et al., 2010a; Hu et al., 2013) and LRO (Smith et al., 2010b, 2011) missions have been used to produce global DTMs respectively. Currently, the LOLA LDEM_1024 is the highest resolution global DTM with a ground spacing of 1024 pixels per degree (ppd) $(30 \mathrm{~m} /$ pixel at the equator) (Smith et al., 2011).

Global DTMs were also produced from stereo orbital images from different missions after block adjustment of the images. The global products include 100m-resolution DTM (GLD100) from LROC WAC images (ASU, 2011a; Scholten et al. 2012), 1024 ppd DTM from 10m-resolution SELENE stereo images (Haruyama et al., 2012), and $20 \mathrm{~m}$-resolution DTM from $7 \mathrm{~m}$ resolution CE-2 stereo images (Li et al., 2015; Ren et al., 2019), and, etc. Recently, Barker et al. (2016) produced a lunar DEM by co-registration and combining SELENE TC DTM with LOLA data; the resultant SLDEM2015 covers latitudes within $\pm 60^{\circ}$ at a horizontal resolution of $512 \mathrm{ppd}$ ( $\sim 60 \mathrm{~m}$ at the equator) and 3-4 m RMS elevation residuals to LOLA profiles.

Subsequently, global DOMs were generated based on the DTMs and the original images with refined orientation parameters. The available global DOMs include $120 \mathrm{~m}$ resolution CE-1 DOM (Li et al., 2010b), 100 m-resolution LROC WAC DOM (ASU, 2011b), 1024 ppd SELENE DOM (Haruyama et al., 2012), and 7 m-resolution Chang'e-2 DOM (Li et al., 2015), etc.

\subsection{Regional and Local Mapping Products}

Meter scale regional and local DTMs and DOMs have been produced using high resolution (meter and sub-meter) orbital images to provide detailed topographic information of the past and future landing sites, and other areas of scientific interests. For example, based on stereo LROC NAC images of resolution up to $0.5 \mathrm{~m}$ acquired from two or more orbits with at least one off-nadir slew, high-resolution DTMs (up to $1.5 \mathrm{~m}$ resolution) and DOMs (up to $0.5 \mathrm{~m}$ resolution) were produced for postmission analyses of Apollo landing sites (e.g., Haase et al., 2012; Karachevtseva et al., 2013), and for characterization of current and future landing sites. The DTMs and DOMs are available through the ASU LROC website (http://wms.lroc.asu.edu/lroc).

In addition to DTM and DOM production from stereo images, high-resolution large regional image mosaics have been produced using single LROC NAC images. For example, USGS produced controlled polar mosaics using NAC images in support of the Lunar Mapping and Modeling Project (Lee et al., 2012; Archinal et al., 2015.). The LROC team produced a Northern Polar Mosaic (LNPM) by assembling 10,581 NAC images; the mosaic is of 681 Gigapixels in size and is the world's largest lunar image mosaic so far (ASU, 2014). 
At the Chang'e-3 and Chang'e-4 lading sites, topographic mapping and analyses have been done at meter to millimeter scales using orbital, descent and rover-based images before launch, after landing and during surface operations (Liu et al., 2014; Wu et al., 2014a, 2020; Di et al., 2019a, 2020). For example, a 4.5 m-resolution DTM and a 1.5 m-resolution DOM of the Chang'e-3 landing site were generated using the $1.5 \mathrm{~m}$ resolution stereo images acquired by Chang'e- 2 and used to support topographic analysis of the landing site. After landing, local DTMs and DOMs with centimeter resolution $(2 \mathrm{~cm}$ to 5 $\mathrm{cm})$ were routinely generated using the stereo images acquired by the rovers' navigation cameras and panoramic cameras to support topographic analysis, hazard recognition, and rover path planning (Liu et al., 2015, 2020). Furthermore, 3-D models of some targets of interest, e.g., rocks and small craters in close range, were produced in millimeter scales to support detailed morphological analysis.

\section{FUTURE PROSPECTS}

Great achievements and progress of lunar topographic mapping have been made in the first two decades of the $21^{\text {st }}$ century based on the unprecedented high-resolution and high-precision data acquired by the successful missions. However, significant challenges remain to make full use of the huge amount of lunar data acquired recently.

First, considerable positioning and elevation discrepancies exist among the mapping products from different missions, or even from the same mission, causing difficulties for synergistic use of the multiple products. This calls for a new high accuracy control network and co-registration techniques for large data sets from multiple missions (Archinalet al., 2006, 2007; Kirk et al., 2012; Di et al., 2017).

Second, a large portion of the new high-resolution data has not been processed for topographic mapping. This requires improvement and utilization of automated systems for topographic mapping using the large data sets more effectively.

Third, merging/fusion of multiple datasets (e.g., images and laser altimetry data) and techniques (e.g., photogrammetry and photoclinometry) is necessary and valuable for lunar topographic mapping and studies (Wu et al., 2013; Liu and Wu, 2020; Barker et al., 2016).

Last but not least, sharing and dissemination of data, topographic mapping techniques, and products are also very important to promote planetary mapping technologies and science applications.

\section{ACKNOWLEDGEMENTS}

This study was supported in part by National Natural Science Foundation of China (Grant no.s 41671458, 41590851 and 41941003).

\section{REFERENCES}

Araki, H., Tazawa, S.T., Noda, H., et al., 2008. Present status and preliminary results of the lunar topography by KaguyaLALT mission. 39th Lunar and Planetary Science Conference, Abstract \#1510.
Araki, H., Tazawa, S.T., Noda, H., et al., 2009. Lunar global shape and polar topography derived from Kaguya-LALT laser altimetry. Science, 323, 897-900.

Archinal, B. A., Rosiek, M. R., Kirk, R. L., et al., 2006. The unified lunar control network 2005. USGS open-file report. http://pubs.usgs.gov/of/2006/1367/ULCN2005-OpenFile.pdf

Archinal, B. A., Rosiek, M. R. Kirk, R. L., et al., 2007. Final Completion of the Unified Lunar Control Network 2005 and Topographic Model. $38^{\text {th }}$ Lunar and Planetary Science Conference, Abstract \#1904.

Archinal, B. A., Lee, E., Weller L, et al., 2015. Status of geodetically controlled high-resolution LROC polar mosaics. 46th Lunar and Planetary Science Conference, Abstract \#1571.

ASU (Arizona State University). 2011a. GLD100 WAC Global DTM. http://wms.lroc.asu.edu/lroc/view_rdr/WAC_GLD100.

ASU. 2011b. WAC Global Morphologic Map. http://wms.lroc.asu.edu/lroc/view_rdr/WAC_GLOBAL.

ASU, 2014. 681 Gigapixels! http://lroc.sese.asu.edu/posts/738.

Barker, M. K., Mazarico, E., Neumann, G. A., et al., 2016. A new lunar digital elevation model from the Lunar Orbiter Laser Altimeter and SELENE Terrain Camera. Icarus, 273, 346-355.

Beyer, R. A., Alexandrov, O., Mcmichael, S., 2018. The Ames stereo pipeline: NASA's open source software for deriving and processing terrain data. Earth and Space Science, 5(9), 537-548.

China Daily, 2019. China's lunar rover travels over 345 meters on moon's far side. http://www.chinadaily.com.cn/a/201912/04/WS5de77085a310c f3e3557bfc4.html

Di, K., Hu, W., Liu, Y., et al. 2012. Co-registration of Chang'E1 stereo images and laser altimeter data with crossover adjustment and image sensor model refinement. Advances in Space Research, 50(12),1615-1628.

Di, K., Liu, Y., Liu, B., et al., 2014. A Self-calibration bundle adjustment method for photogrammetric processing of Chang'E-2 stereo lunar imagery. IEEE Transaction on Geoscience and Remote Sensing, 52(9), 5432-5442.

Di, K., Liu, Z., Wan W. Peng, M., 2015. Lunar and Mars Remote Sensing Mapping and Rover Localization. Science Press, Beijing. (in Chinese)

Di, K., Liu, B., Peng, M., et al., 2017. An initiative for construction of new-generation lunar global control network using multi-mission data, Int. Arch. Photogramm. Remote Sens. Spatial Inf. Sci., XLII-3/W1, 29-34.

Di, K., Liu Z., Liu B., et al, 2019a. Topographic analysis of Chang'e-4 landing site using orbital, descent and ground data. Int. Arch. Photogramm. Remote Sens. Spatial Inf. Sci., XLII2/W13, 1383-1387.

Di, K., Liu, Z., Liu, B., et al. 2019b. Chang'e-4 lander localization based on multi-source data. Journal of Remote Sensing, 23(1): 177-184. 
Di, K., Jia, M., Xin, X., et al., 2019c. High-resolution large-area digital orthophoto map generation using LROC NAC images. Photogrammetric Engineering \& Remote Sensing, 85(7), 481491.

Di, K., Liu, Z., Wan, W., et al., 2020. Geospatial technologies for Chang'e-3 and Chang'e-4 lunar rover missions. Geo-spatial Information Science, 23(1): 87-97.

Foing, B. H., Racca, G. D., Marini, A., et al. 2006. SMART-1 mission to the Moon: Status, first results and goals. Adv Space Res, 37, 6-13.

Greeley, R., Batson, R. M. (eds.) 1990. Planetary Mapping. Cambridge University Press, New York.

Grieger, B., Foing, B. H., Koschny, D., et al., 2008. Coverage and pointing accuracy of SMART-1/AMIE Images. 39th Lunar and Planetary Science Conference, Abstract \#2221.

Goswami, J. N., Annadurai, M., 2009. Chandrayaan-1: India's first planetary science mission to the moon. Curr Sci, 96, 486491.

Haruyama, J., Ohtake, M., Matsunaga, T., et al., 2009. Selene (Kaguya) terrain camera observation results of nominal mission period. $40^{\text {th }}$ Lunar and Planetary Science Conference, Abstract $\# 1553$.

Haruyama, J., Hara, S., Hioki, K., et al., 2012. Lunar global digital terrain model dataset produced from SELENE (Kaguya) terrain camera stereo observations. 43rd Lunar and Planetary Science Conference, Abstract \#1200.

Haase, I., Oberst, J., Scholten, F., et al., 2012. Mapping the Apollo 17 landing site area based on Lunar Reconnaissance Orbiter Camera images and Apollo surface photography. $J$. Geophys. Res. Planets, 117, E00H20.

Haase, I., Gläser, P., and Oberst, J., 2019. Bundle Adjustment of Spaceborne Double-Camera Push-Broom Imagers and Its Application to LROC NAC Imagery, Int. Arch. Photogramm. Remote Sens. Spatial Inf. Sci., XLII-2/W13, 1397-1404.

Henriksen, M.R., Manheim, M.R., Burns, K.N., et al., 2017. Extracting accurate and precise topography from LROC narrow angle camera stereo observations. Icarus, 283, 122-137.

Hirschmuller, H., 2007. Stereo processing by semiglobal matching and mutual information. IEEE Transactions on pattern analysis and machine intelligence, 30(2), 328-341.

$\mathrm{Hu}, \mathrm{H}$., and $\mathrm{Wu}, \mathrm{B}, 2018$. Block Adjustment and coupled epipolar rectification of LROC NAC images for precision Lunar topographic mapping. Planetary and Space Science, 160: 26-38

Hu, H., and Wu, B., 2019. Planetary3D: A photogrammetric tool for 3D topographic mapping of planetary bodies. Int. Arch. Photogramm. Remote Sens. Spatial Inf. Sci., IV-2/W5, 519-526.

Hu, W., Di, K., Liu, Z., et al.,. 2013. A new lunar global DEM derived from Chang'E-1 Laser Altimeter data based on crossover adjustment with local topographic constraint. Planetary and Space Science, 87, 173-182
Jia, Y., Zou, Y., Ping, J., et al., 2018. The scientific objectives and payloads of Chang'E-4 mission. Planetary and Space Science, 162, 207-215.

Karachevtseva, I., Oberst, J., Scholten, F., et al., 2013. Cartography of the Lunokhod-1 landing site and traverse from LRO image and stereotopographic data. Planet. Space Sci., 85, 175-187.

Karachevtseva, I. P., Kokhanov, A. A., Rodionova, J. F., et al., 2016. Mapping of Inner and Outer Celestial Bodies Using New Global and Local Topographic Data Derived From Photogrammetric Image Processing, Int. Arch. Photogramm. Remote Sens. Spatial Inf. Sci., XLI-B4, 411-415,

Kirk, R. L., Archinal, B. A., Gaddis, L. R., and Rosiek, M. R., 2008. Cartography for lunar exploration: 2008 status and mission plans. Int. Arch. Photogramm. Remote Sens. Spatial Inf. Sci., 37(4), 1473-1480.

Kirk, R. L., Cook, D., Howington-Kraus, E., et al., 2010. Radargrammetry with Chandrayaan-1 and LRO Mini-RF images of the Moon: Controlled mosaics and DTMs. European Planetary Science Congress, 5, EPSC2010-703.

Kirk. R. L., Archinal, B. A., Gaddis, L. R., Rosiek, M. R., 2012. Lunar cartography: progress in the 2000s and prospects for the 2010s. Int. Arch. Photogramm. Remote Sens. Spatial Inf. Sci., 39(B4), 489-494.

Kozlova, N., Zubarev, A., Karachevtseva, I., et al., 2014. Some aspects of modern photogrammetric image processing of Soviet lunokhod panoramas and their implemenation for new studies of lunar surface, Int. Arch. Photogramm. Remote Sens. Spatial Inf. Sci., XL-4, 121-126.

Lee, E. M., Weller, L. A., Richie, J. O., et al., 2012. Controlled polar mosaics of the Moon for LMMP by USGS. 43th Lunar and Planetary Science Conference, Abstract \#2507.

Li, C., Ren, X., Liu, J., et al. 2010a. Laser altimetry data of Chang'E-1 and the global lunar DEM model. Sci China Earth Sci, 40(3), 281-293.

Li, C., Liu, J., Ren, X., et al. 2010b. The global image of the moon by the Chang'E-1: Data processing and lunar cartography. Sci China Earth Sci, 40(3), 294-306.

Li, C., Ren, X., Liu, J., et al., 2015. A New Global and High Resolution Topographic Map Product of the Moon from Chang'e-2 Image Data. $46^{\text {th }}$ Lunar and Planetary Science Conference, Abstract \#1638.

Li, R., Wang, W., He, S., et al., 2010. Precision photogrammetric modeling of LROC NAC cameras and topographic products. Annual Meeting of the Lunar Exploration Analysis Group, LPI Contribution No. 1595, 36.

Liu, B., Liu, Y., Di, K., et al., 2014. Block adjustment of Chang'E-1 images based on rational function model. Proceedings of SPIE 9158, Remote Sensing of the Environment: 18th National Symposium on Remote Sensing of China, 91580G.

Liu, B., Xu, B., Di, K., et al., 2016. A Solution to Low RFM Fitting Precision of Planetary Orbiter Images Caused by 
Exposure Time Changing. Int. Arch. Photogramm. Remote Sens. Spatial Inf. Sci., XLI-B4, 441-448.

Liu, B., Jia, M., Di, K., et al. 2018. Geopositioning precision analysis of multiple image triangulation using LROC NAC Lunar images. Planetary and Space Science, 162, 20-30.

Liu, W.C., Wu, B., 2020. An integrated photogrammetric and photoclinometric approach for illumination-invariant pixelresolution 3D mapping of the lunar surface. ISPRS Journal of Photogrammetry and Remote Sensing 159, 153-168.

Liu, Y., and Di, K., 2011. Evaluation of Rational Function Model for Geometric Modeling of Chang'E-1 CCD Images. Int. Arch. Photogramm. Remote Sens. Spatial Inf. Sci., 38(4/W25), 121-125.

Liu, Y., Liu, B., Xu, B., et al., 2014. High Precision Topographic Mapping at Chang'E-3 Landing Site with MultiSource Data. Int. Arch. Photogramm. Remote Sens. Spatial Inf. Sci., XL-4, 157-161.

Liu, Z., Di, K., Peng M., et al, 2015. High precision landing site mapping and rover localization for Chang'e-3 mission. Sci China-Phys Mech Astron, 58(1), 019601.

Liu, Z., Di, K., Li J., et al, 2020. Landing Site topographic mapping and rover localization for Chang'e-4 mission. Science China-Information Sciences, 63, 140901:1-140901:12.

Mazarico, E., Rowlands, D. D., Neumann, G. A., et al., 2012. Orbit determination of the Lunar Reconnaissance Orbiter. Journal of Geodesy, 86(3), 193-207.

Moratto, Z. M., Broxton, M. J., Beyer, R. A., et al., 2010. Ames Stereo Pipeline, NASA's open source automated stereogrammetry software. 41st Lunar and Planetary Science Conference, Abstract \#2364.

Naß, A., Di, K., Elgner, S., et al., 2017. Planetary Cartography And Mapping: Where We Are Today, And Where We Are Heading For? Int. Arch. Photogramm. Remote Sens. Spatial Inf. Sci., XLII-3/W1, 105-112,

Oberst, J., Gwinner, K., and Preusker, F., 2014. Exploration and Analysis of Planetary Shape and Topography Using Stereophotogrammetry. In: T. Spohn, D. Breuer, and T. V. Johnson (Eds.), Encyclopedia of the Solar System, Elsevier, 1223-1233.

Peng, M., Wan, W., Wu, K., et al., 2014. Topographic mapping capbility analysis of Chang'e-3 Navcam stereo images and three-dimensional terrain reconstruction for mission operations. Journal of Remote Sensing, 18(5), 995-1002. (in Chinese with English abstract)

Ping, J., Huang, Q., Yan, J., et al., 2008. Lunar topographic model CLTM-s01 from Chang'E-1 laser altimeter. Science in China Series G: Physics, Mechanics and Astronomy, 38(11): 1601-1612.

Radhadevi, P. V., Solanki, S. S., Nagasubramanian, V., et al., 2013. An algorithm for geometric correction of full pass TMC imagery of Chandrayaan-1. Planetary and Space Science, 79-80: $45-51$
Re, C., Cremonese, G., Dall'asta, E, et al. 2012. Performance evaluation of DTM area-based matching reconstruction of Moon and Mars. Proceedings of the Image and Signal Processing for Remote Sensing XVIII, 8537: 85370V.

Ren, X., Liu, J., Li, C., et al., 2019. A global adjustment method for photogrammetric processing of Chang'E-2 stereo images. IEEE Transactions on Geoscience and Remote Sensing, 57 (9), 6832-6843.

Robinson, M., Brylow, S., Tschimmel, M., et al., 2010. Lunar Reconnaissance Orbiter Camera (LROC) instrument overview. Space Science Reviews, 150, 81-124.

Scholten, F., Oberst, J, Matz, K-D., et al., 2012. GLD100: the near - global lunar $100 \mathrm{~m}$ raster DTM from LROC WAC stereo image data. Journal of Geophysical Research: Planets, 117

Smith, D. E., Zuber, M. T., Neumann, G. A., et al., 2010a. Initial observations from the Lunar Orbiter Laser Altimeter (LOLA). Geophysical Research Letters, 37, L18204.1-6.

Smith, D. E., Zuber, M. T., Jackson, G.B., et al., 2010b. The Lunar Orbiter Laser Altimeter Investigation on the Lunar Reconnaissance Orbiter Mission. Space Science Review, 150, 209-241.

Smith, D., Zuber, M., Neumann, G., et al., 2011. Results from the Lunar Orbiter Laser Altimeter (LOLA): Global, HighResolution Topographic Mapping of the Moon. $42^{\text {nd }}$ Lunar and Planetary Science Conference, abstract \# 2350.

Speyerer, E.J., Wagner, R.V., Robinson, M.S., et al., 2016. Preflight and onorbit geometric calibration of the Lunar Reconnaissance Orbiter Camera. Space Sci. Rev., 200, 357-392.

Tran, T., Howington-Kraus, E., Archinal, B, et al., 2010. Generating Digital Terrain Models from LROC Stereo Images with SOCET SET. $41^{\text {st }}$ Lunar and Planetary Science Conference, Abstract \#2515.

Wan, W., Liu, Z., Di, K., et al., 2014. A Cross-Site Visual Localization Method for Yutu Rover. Int. Arch. Photogramm. Remote Sens. Spatial Inf. Sci., XL-4, 279-284.

Wu, B., Guo, J., Zhang, Y., et al., 2011. Integration of Chang'E1 imagery and laser altimeter data for precision Lunar topographic modeling. IEEE Transactions on Geoscience and Remote Sensing, 49(12), 4889-4903.

Wu, B., Guo, J., Hu, H., et al., 2013. Co-Registration of Lunar Topographic Models Derived from Chang'E-1, SELENE, and LRO Laser Altimeter Data Based on a Novel Surface Matching Method, Earth and Planetary Science Letters, 364, 68-84.

Wu, B., Li, F., Ye, L et al, 2014a. Topographic Modeling and Analysis of the Landing Site of Chang'E-3 on the Moon. Earth and Planetary Science Letters, 405(2014), 257-273.

Wu, B., Hu, H., and Guo, J., 2014b. Integration of Chang'E-2 Imagery and LRO Laser Altimeter Data with a Combined Block Adjustment for Precision Lunar Topographic Modeling. Earth and Planetary Science Letters, 391, 1-15. 
Wu, B., and Liu, W.C., 2017. Calibration of Boresight Offset of LROC NAC Imagery for Precision Lunar Topographic Mapping. ISPRS Journal of Photogrammetry and Remote Sensing, 128, 372-387.

Wu, B., Liu, W. C., Grumpe, A., et al., 2017. Construction of Pixel-Level Resolution DEMs from Monocular Images by Shape and Albedo from Shading Constrained with LowResolution DEM. ISPRS Journal of Photogrammetry and Remote Sensing, 140, 3-19,

Wu, B., Di, K., Oberst, J., and Karachevtseva, I. (eds) 2019. Planetary Remote Sensing and Mapping. CRC Press/Taylor \& Francis Group.

Wu, B., Li, F., Hu, H., et al., 2020. Topographic and Geomorphological Mapping and Analysis of the Chang'E-4
Landing Site on the Far Side of the Moon. Photogrammetric Engineering \& Remote Sensing, 86(4), 247-258.

Xin, X., Liu, B., Di, K., et al., 2018. High-precision coregistration of orbiter imagery and digital elevation model constrained by both geometric and photometric information. ISPRS Journal of Photogrammetry and Remote Sensing, 144, 28-37.

Zubarev, A. E., Nadezhdina, I. E., Kozlova, N. A., et al., 2016. Special Software for Planetary Image Processing and Research, Int. Arch. Photogramm. Remote Sens. Spatial Inf. Sci., XLI-B4, 529-536

Zuo, W., Li, C., and Zhang, Z., 2014. Scientific data and their release of Chang'E-1 and Chang'E-2. Chin. J. Geochem., 33, 24-44. 